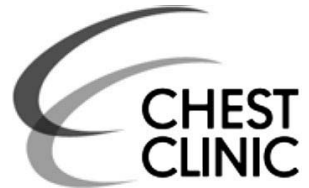

of Medicine, Dentistry and Nursing, NHS Greater Glasgow and Clyde, Glasgow Royal Infirmary, Glasgow, UK ${ }^{2}$ Intensive Care Unit, NHS Greater Glasgow and Clyde, Glasgow Royal Infirmary, Glasgow, UK ${ }^{3}$ Center for Clinical

Management Research, VA Ann Arbor Health System, Ann Arbor, Michigan, USA

${ }^{4}$ Division of Pulmonary and Critical Care, Department of Internal Medicine, University of Michigan, Ann Arbor, Michigan, USA

\section{Correspondence to}

Dr Joanne McPeake, University of Glasgow, School of Medicine, Dentistry and Nursing, NHS Greater Glasgow and Clyde, Glasgow Royal Infirmary, Glasgow, UK;

joanne.mcpeake@glasgow. ac.uk

Received 10 November 2016 Revised 18 January 2017 Accepted 19 January 2017 Published Online First

17 February 2017

\title{
Peer support to improve recovery following critical care discharge: a case-based discussion
}

\author{
Joanne McPeake, ${ }^{1,2}$ Theodore J Iwashyna, ${ }^{3,4}$ Helen Devine, ${ }^{2}$ Pamela MacTavish, ${ }^{2}$ \\ Tara Quasim ${ }^{1,2}$
}

\section{CASE PRESENTATION}

We report the case of a self-employed builder aged 58-years, with a medical history of ischaemic heart disease and type II diabetes. He was transferred to our intensive care unit (ICU) from another local hospital for treatment of gallstone pancreatitis. $\mathrm{He}$ stayed in critical care for 19 days, with a total hospital stay of 9 weeks. He and his wife have consented to the presentation of their case.

This patient required level three care (ICU care) for 3 days. He required level two care (high dependency care) for a further 16 days due to complications related to his acute kidney injury and pancreatitis. He was mechanically ventilated for 3 days with a worst $\mathrm{P} / \mathrm{F}$ ratio of $150 \mathrm{mmHg}$ and underwent renal replacement therapy for 8 days. As per standard practice in the ICU at the time, he was visited by physical therapy on 17 of his 19 ICU days. This patient has two children and a wife who works as a Nursery Teacher. After discharge from hospital, he returned to his own home.

At discharge from hospital, aerobic capacity was assessed using the incremental shuttle walk test. The patient scored a metabolic equivalent of 2.4 on this test (this represents an ability to undertake a low intensity exercise programme). ${ }^{1}$ Grip strength measurements were obtained and were $16 \mathrm{~kg}$ (right hand) and $12 \mathrm{~kg}$ (left hand), less than half of expected when compared with the population norm. ${ }^{2}$

At home, further nutritional support from a nasogastric tube (NGT) was required. The patient also had significant fatigue, limb weakness, lethargy, decreased balance and shortness of breath. At 2 months post-discharge from hospital, neither he nor his wife had returned to work. In light of these problems, at 2 months post-ICU discharge, both the patient and his wife were invited to participate in Intensive Care Syndrome: Promoting Independence and Return to Employment (InS:PIRE).

\section{INTENSIVE CARE SYNDROME: PROMOTING INDEPENDENCE AND RETURN TO EMPLOYMENT}

InS:PIRE is based on the theoretical model of salutogenesis. Salutogenesis, which has as the central component 'sense of coherence', focuses on comprehensibility, manageability and meaningfulness to create and improve health.

InS:PIRE is a short 5-week, person-centred, peer support programme, which aims to empower patients to take control of their health and wellbeing (table 1). Patients and their caregivers co-designed the InS:PIRE programme. ${ }^{4}$ It is a progressive series of meetings, three of which are between one clinician and the patient and caregiver; the final two are group sessions. Each meeting is structured in two parts: a diagnostic phase, to identify problems the patient and caregiver are having based on both review of their ICU and postdischarge medical record as well as interview; and an interventional phase, where individually identified problems are targeted. Due to the frequent and high burden of problems, these diagnostic/interventional meetings are divided into separate domains as shown in table 1 , with frequent co-ordination between clinicians each week to ensure full coverage.

Due to of the high prevalence of weakness and physical disability among critical illness survivors, each week patients receives 1 hour of physiotherapy as a group. This session helps support patients to undertake basic activity around their home. Within InS:PIRE, former patient and caregiver volunteers run an informal discussion. They offer current patients and caregivers the opportunity to discuss their recovery and give information and support from the perspective of someone who has been through similar experiences. They also offer hope for those still experiencing difficulty.

There have been a number of randomised controlled trials (RCTs) showing no population-wide benefit of specific therapeutic approaches in post-ICU work. ${ }^{5}$ InS:PIRE differs from such targeted therapies in several aspects: it includes a Multi-professional diagnostic evaluation, so that personalised and multiple therapies can be brought to bear for any patient, rather than prespecifying a single dominant approach; it occurs much earlier than some of past interventions (eg, in contrast to PRACTICAL's 3-month first contact); it was codesigned with heavy patient and caregiver involvement from the beginning; it is more intensive, involving five consecutive weeks and it includes integrative peer support, pharmacy and social agency involvement, which have not in general been previously tested. ${ }^{6}$ RCT testing of approaches such as InS:PIRE is necessary, but likely several years away from reporting results.

\section{INTERVENTIONS DELIVERED DURING THE INS:PIRE PROGRAMME}

Our patient and his wife suffered from a constellation of problems common among survivors of critical illness. These included muscle weakness, highly disorganised home medication regime, depression 
Table 1 Components of the InS:PIRE programme

\begin{tabular}{lll}
\hline Week & Component & Description \\
\hline 1 & $\begin{array}{l}\text { Pharmacy session } \\
\text { (one to one) }\end{array}$ & $\begin{array}{l}\text { Review current medications, analyse if they } \\
\text { are appropriate and deliver education as } \\
\text { needed }\end{array}$ \\
2 & $\begin{array}{l}\text { Nurse/medical session } \\
\text { (one to one) }\end{array}$ & $\begin{array}{l}\text { Lay summary of ICU offered. Opportunity to } \\
\text { ask any questions. Personal goals are set. } \\
\text { Visit to ICU }\end{array}$ \\
3 & $\begin{array}{l}\text { Physiotherapy session } \\
\text { (one to one) }\end{array}$ & $\begin{array}{l}\text { Physical areas for development are explored. } \\
\text { Issues related to chronic pain are reviewed. } \\
\text { Musculoskeletal problems are assessed and } \\
\text { specific exercise programmes are provided as } \\
\text { required } \\
\text { Explores coping strategies and expected } \\
\text { responses to critical care }\end{array}$ \\
5 & $\begin{array}{l}\text { Psychology session } \\
\text { (group session) } \\
\text { Social prescription } \\
\text { (group session) }\end{array}$ & $\begin{array}{l}\text { housing, carers support and volunteering and } \\
\text { fitness groups }\end{array}$ \\
\hline
\end{tabular}

ICU, intensive care unit; InS:PIRE, Intensive Care Syndrome: Promoting Independence and Return to Employment.

(in both the patient and spouse) and dietary insufficiency. These problems were overlapping and mutually reinforcing; they were also exacerbated by a poor sense of self-efficacy. InS:PIRE seeks to aggressively intervene early to break this cycle, promoting more rapid recovery.

ICU-acquired muscle weakness is poly-factorial and a combined neuromuscular problem lacking targeted therapy, but is quite common even in patients such as those with only 3 days of mechanical ventilation. In order to address the muscle weakness, this patient aged 58 years and his wife attended a weekly exercise class for 5 weeks, which focused specifically on upper and lower limb strengthening, balance re-education and increasing aerobic capacity. He also attended and contributed to five educational discussions based on an ethos of self-management on topics such as the benefits of exercise, pacing and ICU-acquired weakness. A specific strengthening exercise programme was provided to help manage with his muscle weakness. He was keen to continue increasing his fitness levels on discharge from the programme and was referred to his local gym for further rehabilitation.

Our patient's aspirin, atorvastatin, moxonidine, bendrofluazide and perindopril had been held during the hospital admission and not restarted. There was neither any plan made for them to be restarted nor communication as to why they should be permanently discontinued. Previous data suggest that this is not uncommon. ${ }^{7}$ During the pharmacy intervention medicines were reconciled, comparing pre-ICU admission medications, what he received during his ICU stay, and what he was on at the InS:PIRE visit. A letter was sent to the patient's family physician to highlight these omissions and it was recommended that an appointment was made for ongoing blood pressure monitoring. A National Patient Safety Foundation Framework -'Ask Me 3' document was created with the patient regarding the medication issues identified. He was encouraged to use this document as a guide to discuss the above medication changes with his local family physician. A medication passport was completed; this is a booklet that documents his current medications and the reasons for taking them.

Many of the problems of ICU survivorship can only be remediated by active sustained efforts by the patient on his or her own behalf. From our own experience, while there is little about this in the ICU literature, it is common in our population. During the family session with the nurse and medic, a lay summary was provided that documented the ICU stay; the patient was also offered the opportunity to ask any questions, or go over any part of his hospital stay that concerned him. Personal goals to work towards during the InS:PIRE programme were also identified. These focused on improvement in nutrition and removal of the NGT; improvement in his confidence (demonstrated by driving independently) and improvement in balance and removal of the ongoing need for a walking aid. This patient also wanted to return to full-time employment.

Recent data from a caregiver cohort of patients with prolonged mechanical ventilation have demonstrated a high prevalence and enduring mental health difficulties for caregivers. ${ }^{8}$ Our patient's wife also discussed the challenges that she was facing, especially from an emotional perspective. They described that this was the first time that they had spoken openly to one another about the experience of ICU and the difficult recovery trajectory which they faced. As a result, the couple pledged to have a holiday together.

Other interventions which the couple received during the InS: PIRE programme included group sessions with psychology and a social prescription session. During the social prescription week, local community resources to help with recovery were discussed with the couple. These included support for dietary advice and information on obtaining a disability permit for driving. The couple also spoke with other patients and caregivers who were at a similar stage in their recovery as well as the patient volunteers who were also involved in delivering the programme objectives.

\section{OUTCOMES}

By the end of the 5-week InS:PIRE programme, the patient's NGT was removed and he was able to provide his own nutrition by eating little and often. He was also driving wherever he wanted independently. He felt more confident, as evidenced by his ability to undertake the exercises from the personalised physiotherapy session. Furthermore, by the end of the 5 -week programme, he no longer needed the walking aid and as a result of his improved exercise capacity he no longer required the disabled parking badge. At the 6-month follow-up appointment, both the patient and his caregiver had returned to full-time employment and at 1 year, the couple had been on holiday together.

Qualitative feedback from the patient about the programme included 'There was clarification of procedures undergone and opportunity to ask questions. It was an acceleration to recovery'. Our patient's wife also gave feedback that included 'it gave me the opportunity to voice hidden fears and thoughts'.

\section{DISCUSSION}

Patients and caregivers recovering from a critical illness may experience significant financial, social and physical challenges in the months and years after discharge, yet there have been few tested (and fewer proven successful) remediation programmes. The InS:PIRE programme offers an integrated package of mutually reinforcing recovery programmes that are not widely available. In the absence of InS:PIRE, the use of peer support groups for survivors may be a low cost alternative, as might efforts to provide some or all of the individual pieces via individual referrals. It is unknown to what extent there are beneficial interactions to synchronous provision of all services-but pending such rigorous evaluation, InS:PIRE may also provide a menu of potential interventions that clinicians should consider.

Twitter Follow Joanne McPeake @jomcpeake22, Pamela MacTavish @pamelamactavish, Tara Quasim @taraquasim and Theodore Iwashyna @iwashyna 
Contributors All authors have read and approved the manuscript.

Funding JMCP is supported by a CNO Scotland Fellowship and the Health Foundation. TJI work was supported, in part, by the US Department of Veterans Affairs, Health Services Research \& Development, IIR 13-079. TQ, PMacT and HD are supported by the Health Foundation.

Disclaimer This work does not necessarily represent the views of the US Government or Department of Veterans Affairs.

Competing interests None declared.

Provenance and peer review Not commissioned; externally peer reviewed.

\section{REFERENCES}

1 Jetté M, Sidney K, Blümchen G. Metabolic equivalents (METS) in exercise testing, exercise prescription and evaluation of functional capacity. Clin Cardiol 1990;13:555-65.
2 Corner EJ, Wood H, Englebretsen CE, et al. The Chelsea Critical Care Physical Assessment Tool (CPAx): validation of an innovative new tool to measure physical morbidity in the general adult critical care population; an observational proof-of-concept pilot study. Physiotherapy 2013;99:33-41.

3 Antonovsky A. Health, stress and coping. San Francisco: Jossey-Bass Publishers, 1979.

4 McPeake J, Sherriff-Short M, Smart G, et al. The creation of the first ICU patient and family advisory council in the UK. Crit Care Med 2015;43:225-6.

5 Connolly B, Salisbury L, O'Neill B, et al. for the ERACIP Group. Exercise rehabilitation following intensive care unit discharge for recovery from critical illness. Cochrane Database Syst Rev 2015;(6):CD008632.

6 Cuthbertson BH, Rattray J, Campbell MK, et al. The PRaCTCaL study of nurse led, intensive care follow-up programmes for improving long term outcomes from critical illness: a pragmatic randomised controlled trial. BMJ 2009:339:B3723.

7 Eijsbroek H, Howell DCJ, Smith F, et al. Medication issues experienced by patients and carers after discharge from the intensive care unit. J Crit Care 2013:28:46-50.

8 Cameron Jl, Chu LM, Matte A, et al. One-year outcomes in caregivers of critically ill patients. N Engl J Med 2016;374:1831-41. 